The Journal of $\mathbf{N}_{\text {ondinear }} \mathbf{S}_{\text {cience and Applications }}$ http://www.tjnsa.com

\title{
SEPARATION THEOREM WITH RESPECT TO SUB-TOPICAL FUNCTIONS AND ABSTRACT CONVEXITY
}

\author{
M. ALIMOHAMMADY ${ }^{1,2}$ AND A. SHAHMARI ${ }^{2, *}$ \\ Communicated by S. M. Vaezpour
}

\begin{abstract}
This paper deals with topical and sub-topical functions in a class of ordered Banach spaces. The separation theorem for downward sets and sub-topical functions is given. It is established some best approximation problems by sub-topical functions and we will characterize sub-topical functions as superimum of elementary sub-topical functions.
\end{abstract}

\section{INTRODUCTION AND PRELIMINARIES}

Topical functions are intensively studied (see $[2,3]$ ), and they have many applications in various parts of applied mathematics in particular in the modeling of discrete event system (see [2,3]). Topical functions are also interesting from a different point of view, namely as a tool in the study of the so-called downward sets. Downward set arise in the study of some problems of mathematical economics and game theory (see [4]).

Moreover, topical functions have studied in much more general class of subtopical (increasing plus-sub-homogeneous) functions (see [9]). In section 1, we recall some definitions and establish some results related to topical functions of $\varphi(x, y):=\sup \{\lambda \in \mathbb{R}: \lambda \cdot 1 \leq x+y\}$. In section 2 , we will prove some basic properties of sub-topical functions, we prove separability theorem for downward sets and sub-topical functions. In section 3, it is given other form of separation theorem with respect to sub-topical function. We would characterize best approximation problem by sub-topical functions.It is given separation theorem for

Date: Received: 15 January 2009, Revised: 18 April 2009.

* Corresponding author.

2000 Mathematics Subject Classification. Primary 35P20, Secondary 35J70; 35BJ20.

Key words and phrases. Downward set, Subdifferential set, ordered Banach space, topical function, sub-topical function, separation theorem, abstract convex set. 
downward sets and sub-topical functions. Finally we establish best approximation problem by sub-topical functions and characterize sub-topical functions as superimum of elementary sub-topical functions.

Let $(X,\|\|$.$) be a Banach space and C$ be a closed convex cone in $X$ such that $C \cap(-C)=\{0\}$ and $\operatorname{int} C \neq \emptyset . X$ is equipped with the relation $\geq$ i.e; generated by $C: y \leq x$ if and only if $x-y \in C(x, y \in X)$ and $C: y<x$ if and only if $x-y \in \operatorname{int} C(x, y \in X)$. Assume that $C$ is a normal cone. Recall that a cone $C$ is called normal if there exits a constant $m>0$ such that $\|x\| \leq m\|y\|$, whenever $0 \leq x \leq y$ and $x, y \in X$. Let $\mathbf{1} \in$ int $C$ and

$$
B=\{x \in X: \mathbf{- 1} \leq x \leq \mathbf{1}\} \text {. }
$$

It is well known and easy to check that $B$ can be considered as the unit ball under a certain norm $\|.\|_{1}$, which is equivalent to the initial norm $\|$.$\| . Without loss of$ generality one can assume that $\|\cdot\|=\|\cdot\|_{1}$ (see [6]).

For any subset $W$ of $X$, denote by int $W, c l W$ and $b d W$ the interior, closure and boundary of $W$ respectively.

For a non-empty subset $W$ of $X$ and $x \in X$, define ( see[6])

$$
d(x, W)=\inf _{w \in W}\|x-w\| .(2)
$$

Recall (see [6]), a point $w_{o} \in W$ is called best approximation for $x \in X$, if

$$
\left\|x-w_{o}\right\|=d(x, W) .
$$

Let $W \subset X$. For $x \in X$ it is denoted by $P_{W}(x)$ the set of all best approximations of $x$ in $W$ :

$$
P_{W}(x)=\{w \in W:\|x-w\|=d(x, W)\} .
$$

It is well-known that $P_{W}(x)$ is a closed and bounded subset of $X$. If $x \in X \backslash W$ then $P_{W}(X)$ is located in the boundary of $W$ (see $[6]$ ).

For $x \in X$ and $r>0$, according to (1),

$$
B(x, r):=\{y \in X:\|x-y\| \leq r\}=\{y \in X: x-r \cdot \mathbf{1} \leq y \leq x+r \cdot \mathbf{1}\} .
$$

Definition 1.1. [5, 6] A function $f: X \longrightarrow \overline{\mathbb{R}}$ to the set of extended real numbers is a topical function if

a) (Plus-homogeneous), i.e, $f(x+\lambda . \mathbf{1})=f(x)+\lambda$ for $\forall x \in X$ and $\forall \lambda \in \mathbb{R}$,

b) (Increasing function), i.e; if $x \leq y$ then $f(x) \leq f(y)$.

Let $\varphi: X \times X \longrightarrow \mathbb{R}$ be a function which is defined by

$$
\varphi(x, y):=\sup \{\lambda \in \mathbb{R}: \lambda \cdot \mathbf{1} \leq x+y\}(\forall x, y \in X) .
$$

From (5) it is easy to see that the set $\{\lambda \in \mathbb{R}: \lambda \cdot \mathbf{1} \leq x+y\}$ is non-empty and bounded above by $\|x+y\|$. Clearly this set is closed. It follows from the definition of $\varphi \varphi$ enjoys the following properties:

$$
-\infty<\varphi(x, y) \leq\|x+y\| \text { for all } x, y \in X .(6)
$$




$$
\begin{gathered}
\varphi(x, y) \cdot \mathbf{1} \leq x+y \text { for all } x, y \in X .(7) \\
\varphi(x, y)=\varphi(y, x) \text { for all } x, y \in X .(8) \\
\varphi(x,-x)=\sup \{\lambda \in \mathbb{R}: \lambda \cdot \mathbf{1} \leq x-x=0\}=0 \text { for all } x \in X .(9)
\end{gathered}
$$

For each $y \in X$, define a function $\varphi_{y}: X \longrightarrow \mathbb{R}$ by

$$
\varphi_{y}(x):=\varphi(x, y) \forall x \in X .(10)
$$

Let $f: X \longrightarrow \mathbb{R}$. Recall that directional derivative $f_{+}^{\prime}(x, u)$ of $f$ at $x \in X$ in direction of $u \in X$ is defined by (see [7]),

$$
f_{+}^{\prime}(x, u):=\lim _{t \longrightarrow 0^{+}} \frac{f(x+t u)-f(x)}{t} .
$$

\section{BASIC PROPERTIES OF SUB-TOPICAL FUNCTIONS}

Definition 2.1. 9] A function $f: X \longrightarrow \overline{\mathbb{R}}$ is called plus-sub-homogeneous if

$$
f(x+\lambda . \mathbf{1}) \leq f(x)+\lambda \forall x \in X \text { and } \forall \lambda \in \mathbb{R}_{+} .
$$

$f$ is called sub-topical if it be increasing and plus-sub-homogeneous.In the following, we characterize plus-sub-homogenous which its proof is direct.

Lemma 2.2. A function $f: X \longrightarrow \overline{\mathbb{R}}$ is plus-sub-homogeneous if and only if

$$
f(x+\lambda .1) \geq f(x)+\lambda \forall x \in X \text { and } \forall \lambda \in \mathbb{R}_{-} .
$$

Let us present some examples of sub-topical functions:

\section{Example 2.3. .}

a) Every topical function is sub-topical.

b) Every sub-linear function such that $f(\mathbf{1}) \leq 1$ is sub-topical. Indeed

$$
f(x+\lambda . \mathbf{1}) \leq f(x)+\lambda f(\mathbf{1}) \leq f(x)+\lambda \forall x \in X \text { and } \forall \lambda \in \mathbb{R}_{+} .
$$

Following is a result for showing Lipschitz continuity of a sub-topical function. Its proof is direct.

Theorem 2.4. Let $f: X \longrightarrow \mathbb{R}$ be a sub-topical function, then $f$ is Lipschitz continuous.

Theorem 2.5. Let $f: X \longrightarrow \overline{\mathbb{R}}$ be a sub-topical function. Then the following assertions are true.

a) If there exists $x \in X$ such that $f(x)=\infty$, then $f \equiv \infty$.

b) If there exists $x \in X$ such that $f(x)=-\infty$, then $f \equiv-\infty$. 


\section{Proof.}

a) Suppose that there exists $x \in X$ such that $f(x)=\infty$. Let $y \in X$. $\lambda=\varphi(-x, y)$. There are two cases:

Case (1): If $\lambda<0$, by (7) we have $\varphi(-x, y) \cdot \mathbf{1} \leq y-x$, then $x \leq y-\lambda \cdot \mathbf{1}$. Since $-\lambda>0$ and $f$ is sub-topical, so

$$
f(x) \leq f(y)-\lambda .
$$

It implies that $f(y)=\infty$.

Case (2): If $\lambda \geq 0$, then $0 \leq \varphi(-x, y) \cdot \mathbf{1} \leq y-x$, so $x \leq y$ and $f$ is an increasing. Then $f(x) \leq f(y)$, so $f(y)=\infty$.

b) Let $y \in X$ be an arbitrary, $\lambda=\varphi(x,-y)$. Then the remind of proof is similar to that one in $(a)$.

Theorem 2.6. Let $f: X \longrightarrow \overline{\mathbb{R}}$ be an increasing function. Then $f$ is plus-subhomogeneous if and only if,

$f_{x}: \mathbb{R}_{+} \longrightarrow \overline{\mathbb{R}}$ given by $f_{x}(\alpha)=f(x+\alpha \cdot \mathbf{1})-\alpha$, is decreasing.

Proof. If $f(x)=\infty$ (or, $-\infty$ ) for some $x \in X$, by theorem $2.5 f \equiv \infty$ (or, $-\infty$ ), then $f_{x} \equiv \infty(-\infty)$ and so $f_{x}$ is decreasing for all $x \in X$. Therefore, $f: X \longrightarrow \mathbb{R}$ is sub-topical and $0 \leq \alpha \leq \beta$.

$$
\begin{gathered}
f_{x}(\beta)=f(x+\beta \cdot \mathbf{1})-\beta=f(x+\alpha \cdot \mathbf{1}+(\beta-\alpha) \cdot \mathbf{1})-\beta \\
\leq f(x+\alpha \cdot \mathbf{1})+\beta-\alpha-\beta=f_{x}(\alpha) .
\end{gathered}
$$

Conversely, if $f_{x}$ is decreasing, $f_{x}(0) \geq f_{x}(\alpha)$ for all $\alpha \geq 0$. Hence, $f(x) \geq$ $f(x+\alpha \cdot \mathbf{1})-\alpha$ and $f$ is plus-sub-homogeneous.

Theorem 2.7. Let $f: X \longrightarrow \mathbb{R}$ be an increasing function. Then $f$ is plus-subhomogeneous if and only if $f_{+}^{\prime}(x, 1) \leq 1(\forall x \in X)$.

Proof. $(\Longrightarrow)$. According to theorem 2.6, $f_{x}$ is decreasing. Then $\left(f_{x}\right)_{+}^{\prime}(\lambda) \leq$ $0(\forall x \in X)$.

$$
\begin{gathered}
\left(f_{x}\right)_{+}^{\prime}(\lambda)=\lim _{t \longrightarrow 0^{+}} \frac{f_{x}(\lambda+t)-f_{x}(\lambda)}{t} \\
\lim _{t \rightarrow 0^{+}} \frac{f(x+\lambda \cdot \mathbf{1}+t \cdot \mathbf{1})-\lambda-t-f(x+\lambda \cdot \mathbf{1})+\lambda}{t} .
\end{gathered}
$$

If $z:=x+\lambda \cdot \mathbf{1}$

$$
\left(f_{x}\right)_{+}^{\prime}(\lambda)=\lim _{t \longrightarrow 0^{+}} \frac{f(z+t \cdot \mathbf{1})-t-f(z)}{t}=\left(f_{z}\right)_{+}^{\prime}(0)=f_{+}^{\prime}(z, \mathbf{1})-1 .
$$

Therefore, $f_{+}^{\prime}(x, \mathbf{1}) \leq 1, \forall x \in X$.

$(\Longleftarrow)$ Conversely, if $f_{+}^{\prime}(x, 1) \leq 1$ for all $x \in X$, then $\left(f_{x}\right)_{+}^{\prime}(\lambda) \leq 0$. Indeed $\left(f_{x}\right)_{+}^{\prime}(\lambda)=f_{+}^{\prime}(x+\lambda \cdot \mathbf{1}, \mathbf{1})-1$. Therefore, $f_{x}$ is decreasing and $f$ is plus-subhomogeneous. 
Let $\left\{f_{\alpha}: \alpha \in I\right\}$ be a family of sub-topical functions. Then $\bar{f}(x)=\sup _{\alpha \in I} f_{\alpha}(x)$ and $\underline{f}(x)=\inf _{\alpha} f_{\alpha}(x)$ are sub-topical functions.

Next result is an example for a sub-topical function which is not topical.

Example 2.8. If $\alpha>1$, we define

$$
\varphi_{\alpha}(x, y):=\sup \{\lambda \in \mathbb{R}: \lambda \alpha \cdot \mathbf{1} \leq x+y\}(\forall x, y \in X)
$$

It follows from (14) that the set $\{\lambda \in \mathbb{R}: \lambda \alpha \cdot \mathbf{1} \leq x+y\}$ is non-empty and bounded from above $\left(\right.$ by $\left.\alpha^{-1}\|x+y\|\right)$. Clearly this set is closed. It follows from the definition of $\varphi_{\alpha}$ that $\varphi_{\alpha}$ enjoys the following properties:

$$
\begin{gathered}
-\infty<\varphi_{\alpha}(x, y) \leq \alpha^{-1}\|x+y\| \text { for all } x, y \in X .(15) \\
\varphi_{\alpha}(x, y) \cdot \mathbf{1} \leq \alpha^{-1}(x+y) \text { for all } x, y \in X .(16) \\
\varphi_{\alpha}(x, y)=\varphi_{\alpha}(y, x) \text { for all } x, y \in X .(17) \\
\varphi_{\alpha}(x,-x)=\sup \{\lambda \in \mathbb{R}: \lambda \alpha \cdot \mathbf{1} \leq x-x=0\}=0 \text { for all } x \in X .
\end{gathered}
$$

For each $y \in X$ define the function $\varphi_{\alpha, y}: X \longrightarrow \mathbb{R}$ by

$$
\varphi_{\alpha, y}(x):=\varphi_{\alpha}(x, y) \forall x \in X .
$$

Then,

$$
\varphi_{\alpha, y}(x)=\varphi_{\alpha}(x, y)=\alpha^{-1} \varphi(x, y)=\alpha^{-1} \varphi_{y}(x) .
$$

Lemma 2.9. Let $\varphi_{\alpha}$ be the function defined by (14). Then

a) For $1 \leq \alpha \leq \beta$, then $\varphi_{\beta} \leq \varphi_{\alpha} \leq \varphi$.

b) $\lim _{\alpha \longrightarrow 1^{+}} \varphi_{\alpha}(x, y)=\sup _{\alpha>1} \varphi_{\alpha}(x, y)=\varphi(x, y)$

Proof. (a). $\varphi_{\beta}=\beta^{-1} \varphi \leq \alpha^{-1} \varphi=\varphi_{\alpha}$ (b). $\lim _{\alpha \longrightarrow 1^{+}} \varphi_{\alpha}(x, y)=\lim _{\alpha \longrightarrow 1^{+}} \alpha^{-1} \varphi(x, y)=\varphi(x, y)$.

Consider $X_{\varphi_{\alpha}}=\left\{\varphi_{\alpha, y}: \alpha>1, y \in X\right\}$. Lemma 2.2 shows that, elements of $X_{\varphi_{\alpha}}$ can be elementary function for $\varphi$, (i.e; $\varphi_{y}(x)=\sup \left\{\varphi_{\alpha, y}(x): \varphi_{\alpha, y} \in X_{\varphi_{\alpha}}\right\}$ ).

Remark.1. The function $\varphi_{\alpha, y}$ defined by (19) is sub-topical, so by theorem 2.4 is Lipschitz continuous.

Now it is given a characterization of downward sets in terms of separation from outside points by sub-topical functions instead of topical functions. 
Theorem 2.10. Let $\varphi_{\alpha}$ be the function defined by (14). Then for a nonempty subset $W$ of $X$ the following assertions are equivalent:

i) $W$ is a downward subset of $X$.

ii) For each $x \in X \backslash W$,

$$
\varphi_{\alpha}(w,-x)<0, \quad(\forall w \in W) .(20)
$$

iii) For each $x \in X \backslash W$, there exists $l \in X$ such that

$$
\varphi_{\alpha}(w, l)<0 \leq \varphi_{\alpha}(x, l), \quad(\forall w \in W) .
$$

Proof. $(i) \Longrightarrow(i i)$. Suppose that $(i)$ holds and there exists $x \in X \backslash W$. It is known (in [6]) that, $\varphi(w,-x)<0$. Therefore, $\varphi_{\alpha}(w,-x)=\alpha^{-1} \varphi(w,-x)<$ $0(\forall w \in W)$.

(ii) $\Longrightarrow$ (iii). Assume that (ii) holds and $x \in X \backslash W$ is arbitrary. Then by hypothesis, $\varphi_{\alpha}(w,-x)<0(\forall w \in W)$. Let $l=-x \in X$,

$$
\varphi_{\alpha}(w,-x)=\varphi_{\alpha}(w, l)<0=\varphi_{\alpha}(x,-x)=\varphi_{\alpha}(x, l) .
$$

(iii) $\Longrightarrow($ i). Suppose that (iii) holds and $\mathrm{W}$ is not downward set. There is $x \leq w$ such that $w \in W$ and $x \in X \backslash W$. There is $l \in X$ such that $\forall \alpha>1, \varphi_{\alpha}(w, l)<$ $0 \leq \varphi_{\alpha}(x, l)$. But $\varphi_{\alpha, l}(\cdot)$ is increasing. Therefore,

$$
\varphi_{\alpha}(x, l) \leq \varphi_{\alpha}(w, l)<0,
$$

which is a contradiction.

Theorem 2.11. [6] For a function $f: X \longrightarrow \overline{\mathbb{R}}$, the following assertions are equivalent:

i) $f$ is topical.

ii) For each $y \in X$, there exists $l_{y} \in X$ such that

$$
\varphi_{l_{y}}: X \longrightarrow \mathbb{R}
$$

satisfies in

$$
\varphi_{l_{y}} \leq f \text { and } f(y)=\varphi_{l_{y}}(y)(y \in \operatorname{dom} f) .
$$

iii) $f$ in $X_{\varphi}-$ convex.

Example 2.12. Let the function $f: \mathbb{R} \longrightarrow \mathbb{R}$ given by $f(x)=x$ is topical and for $\alpha=2, \varphi_{2}: \mathbb{R} \times \mathbb{R} \longrightarrow \mathbb{R}$ is defined by

$$
\varphi_{2}(x, y)=2^{-1}(x+y)(\forall x, y \in X) .
$$

For arbitrary but fixed $y \in \mathbb{R}$, if there exist $l_{y} \in \mathbb{R}$ such that

$$
\varphi_{2, l_{y}}(x) \leq f(x) \forall x \in \mathbb{R} \text { and } \varphi_{2, l_{y}}(y)=f(y),
$$

then $l_{y}=y$ and $y \leq x \forall x \in \mathbb{R}$ which is a contradiction. 


\section{Separation theorem and abStract CONVEXity by Sub-TOpiCAL FUNCTIONS}

Remark.2. Define, $S_{y, d}(\cdot):=\min \left\{\varphi_{y}(\cdot), d\right\}$ if $y \in X$ and $d \in \mathbb{R}$, then $S_{y, d}$ is sub-topical. Indeed $S_{y, d}$ is increasing since $\varphi_{y}$ is increasing. Also

$$
\begin{aligned}
& (*) y_{1} \leq y_{2} \Longleftrightarrow S_{y_{1}, d} \leq S_{y_{2}, d} \\
& (* *) d_{1} \leq d_{2} \Longleftrightarrow S_{y, d_{1}} \leq S_{y, d_{2}} .
\end{aligned}
$$

and if $x \in X, \lambda>0$

$$
\begin{gathered}
S_{y, d}(x+\lambda \cdot \mathbf{1})=\min \left\{\varphi_{y}(x+\lambda \cdot \mathbf{1}), d\right\}=\min \left\{\varphi_{y}(x)+\lambda, d=d_{1}+\lambda\right\} \\
=\min \left\{\varphi_{y}(x), d_{1}\right\}+\lambda=S_{y, d_{1}}(x)+\lambda \leq S_{y, d}(x)+\lambda .
\end{gathered}
$$

Theorem 3.1. Let $f: X \longrightarrow \overline{\mathbb{R}}$ be a function. The following assertions are equivalent:

i) $f$ is topical.

ii) $f(x) \geq S_{y, d}(x)+f(-y)$ for all $x, y \in X, d \in \mathbb{R}_{+}$.

Proof. Suppose that (i) holds and since $S_{y, d}(x)=\min \{\varphi(x, y), d\} \leq \varphi_{y}(x)$. Then by (see [1]),

$$
S_{y, d}(x) \leq \varphi_{y}(x) \leq f(x)-f(-y)
$$

It implies that

$$
f(x) \geq S_{y, d}(x)+f(-y) .
$$

Conversely, assume that (ii) holds, if $f(x)=\infty($ or $-\infty)$ for some $x \in X$, by hypothesis $f \equiv \infty($ or $-\infty)$. Then $f$ is topical. We assume that $f: X \longrightarrow \mathbb{R}$.

$$
f(x+\lambda \cdot \mathbf{1}) \geq S_{-x,|\lambda|}(x+\lambda \cdot \mathbf{1})+f(x) .
$$

By definition of $S_{y, d} \in S$,

$$
f(x+\lambda \cdot \mathbf{1}) \geq f(x)+\lambda(I)
$$

and

$$
f(x)+\lambda \geq S_{-x-\lambda \cdot \mathbf{1},|\lambda|}(x)+f(x+\lambda \cdot 1)+\lambda=f(x+\lambda \cdot \mathbf{1})(I I)
$$

Therefore, $(I)$ and $(I I)$ imply that $f(x+\lambda \cdot \mathbf{1})=f(x)+\lambda$. We show that $f$ is increasing. Let $x \leq y$ for $x, y \in X$. According to (9):

$$
0=\varphi(x,-x) \leq \varphi(y,-x)
$$

and

$$
0=S_{-x, 0}(y)=\min \left\{\varphi_{-x}(y), 0\right\} \leq \varphi_{-x}(y) \leq f(y)-f(x)
$$

Therefore, $f(x) \leq f(y)$.

Theorem 3.2. The map $\xi: X \times \mathbb{R} \longrightarrow S=\left\{S_{y, d}: y \in X, d \in \mathbb{R}\right\}$ which $(y, d) \mapsto S_{y, d}$ is bijection. 
Proof. $\xi$ is obviously onto. We show $f$ is one-to-one. If $S_{y_{1}, d_{1}}=S_{y_{2}, d_{2}}$, $S_{y_{1}, d_{1}}\left(-y_{1}+d_{1} \cdot \mathbf{1}\right)=d_{1}=S_{y_{2}, d_{2}}\left(-y_{1}+d_{1} \cdot \mathbf{1}\right)=\min \left\{\varphi\left(y_{2},-y_{1}\right)+d_{1}, d_{2}\right\}$. Then $d_{1} \leq d_{2}$ and $d_{1} \leq \varphi\left(y_{2},-y_{1}\right)+d_{1}$, so $0 \leq \varphi\left(y_{2},-y_{1}\right)$ and implies that $0 \leq \varphi\left(y_{2},-y_{1}\right) \cdot \mathbf{1} \leq y_{2}-y_{1}$, so $y_{1} \leq y_{2}$. Also since, $S_{y_{2}, d_{2}}\left(-y_{2}+d_{2} \cdot \mathbf{1}\right)=$ $d_{2}=S_{y_{1}, d_{1}}\left(-y_{2}+d_{2} \cdot \mathbf{1}\right)=\min \left\{\varphi\left(y_{1},-y_{2}\right)+d_{2}, d_{1}\right\}$, then $d_{2} \leq d_{1}$ and $d_{2} \leq$ $\varphi\left(y_{1},-y_{2}\right)+d_{2}$, so $0 \leq \varphi\left(y_{1},-y_{2}\right)$ and implies that $0 \leq \varphi\left(y_{1},-y_{2}\right) \cdot \mathbf{1} \leq y_{1}-y_{2}$. Therefore, $y_{2} \leq y_{1}$. It follows that $y_{1}=y_{2}$ and $d_{1}=d_{2}$.

Theorem 3.3. Let $f: X \longrightarrow \overline{\mathbb{R}}$ be a function. If $f$ is a topical function then there exists a set $M=Y \times \mathbb{R}_{+} \subseteq X \times \mathbb{R}$ such that

$$
f(x)=\sup _{(y, d) \in M} S_{y, d}(x)
$$

In this case, one can take $Y=\{y \in X: f(-y) \geq 0\}$.

Proof. Let $f$ be a topical. If $f(x)=\infty$ for some $x \in X$, by theorem (3.6), $f \equiv \infty$. Then $Y=X$, so $f(x)=\sup _{y \in Y} \varphi(x, y)=\infty$. If $f(x)=-\infty$ for some $x \in X$, by theorem 2.5, $f \equiv-\infty$. Then $Y=\emptyset$ and $f(x)=\sup _{y \in Y} \varphi(x, y)=-\infty$. Suppose that $f: X \longrightarrow \mathbb{R}$, be a topical function. According to theorem 2.11, $\forall x \in X$ there exists $y \in Y$ such that,

$$
\varphi_{y} \leq f, \varphi_{y}(x)=f(x) .
$$

Choose $d=|f(x)|, S_{y, d}=\min \left\{\varphi_{y}, d\right\} \leq f$ and $S_{y, d}(x)=f(x)$. Therefore, $f(x)=\sup _{(y, d) \in M} S_{y, d}(x)$.

Definition 3.4. The lower polar-function of $f: X \longrightarrow \overline{\mathbb{R}}$ is the function $f^{\star}$ : $S \longrightarrow \overline{\mathbb{R}}$

$$
f^{\star}\left(S_{y, d}\right):=\sup _{x \in X}\left\{S_{y, d}(x)-f(x)\right\}, \quad\left(\forall S_{y, d} \in S\right) .
$$

Theorem 3.5. Let $f: X \longrightarrow \overline{\mathbb{R}}$ be a function, then

$$
f^{\star}\left(S_{y, d}\right) \geq d-f(-y+d \cdot \mathbf{1})\left(\forall S_{y, d} \in S\right) .
$$

$f$ is topical if and only if

$$
f^{\star}\left(S_{y, d}\right)=-f(-y)\left(\forall S_{y, d} \in S\right) .
$$

Proof. $f^{\star}\left(S_{y, d}\right)=\sup _{x \in X}\left\{S_{y, d}(x)-f(x)\right\}$ $\geq S_{y, d}(-y+d \cdot \mathbf{1})-f(-y+d \cdot \mathbf{1})=d-f(-y+d \cdot \mathbf{1})$. Indeed $S_{y, d}(-y+d \cdot \mathbf{1})=d$. Then $f^{\star}\left(S_{y, d}\right) \geq d-f(-y+d \cdot \mathbf{1})$. If $f$ is a topical function. Let $x, y \in X$ be arbitrary. It follows from (7) that $S_{y, d}(x) \cdot \mathbf{1} \leq x+y$ and hence $S_{y, d}(x) \cdot \mathbf{1}-y \leq x$. Since $f$ is topical function,

$$
S_{y, d}(x)-f(x) \leq-f(-y)(x, y \in X) \text {. }
$$

Then

$$
f^{\star}\left(S_{y, d}\right)=\sup _{x \in X}\left\{S_{y, d}(x)-f(x)\right\} \leq-f(-y),(y \in Y) .
$$


From $(24), d-f(-y+d \cdot \mathbf{1})=d-f(-y)-d=-f(-y) \leq f^{*}\left(S_{y, d}\right) \leq-f(-y)$. Therefore, $f^{\star}\left(S_{y, d}\right)=-f(-y)$.

Conversely, we assume that (25) holds. Let $x, y \in X$ be arbitrary. $f^{\star}\left(S_{y, d}\right) \geq$ $S_{y, d}(x)-f(x)$. By $(25),-f(-y) \geq S_{y, d}(x)-f(x)$, so $f(x) \geq S_{y, d}(x)+f(-y)$. By theorem 3.3, $f$ is a topical function which it completes the proof.

Definition 3.6. Let $f: X \longrightarrow \overline{\mathbb{R}}$ be a topical function and $S_{l, d} \in S$. Define the $X_{s}-$ subdifferential $\partial_{X_{s}} f(y)$ of at a point $y \in X$ by,

$$
\partial_{X_{s}} f(y)=\left\{(l, d) \in X \times \mathbb{R}: S_{l, d}(x) \leq f(x) \forall x \in X, \text { and } S_{l, d}(y)=f(y)\right\},
$$

where $X_{s}:=\left\{(l, d) \in X \times \mathbb{R}: S_{l, d} \in S\right\}$.

Theorem 3.7. Let $f: X \longrightarrow \mathbb{R}$ be a topical function and $y \in X$. Then

$$
\partial_{X_{s}} f(y)=\left\{(l, d) \in X \times \mathbb{R}: S_{l, d}(y) \geq f(y) \text { and } f(-l)=0\right\} .
$$

In particular, $(f(y) \cdot \mathbf{1}-y, f(y)) \in \partial_{X_{s}} f(y)$ and $(f(y) \cdot \mathbf{1}-y,|f(y)|) \in \partial_{X_{s}} f(y)$

Proof. Let

$$
Q:=\left\{(l, d) \in X \times \mathbb{R}: S_{l, d}(y) \geq f(y) \text { and } f(-l)=0\right\} .
$$

Let $(l, d) \in \partial_{X_{s}} f(y)$. Then $f(y) \leq S_{l, d}(y)$. It follows that $f(y) \cdot \mathbf{1} \leq S_{l, d}(y) \cdot \mathbf{1} \leq$ $\varphi_{l}(y) \cdot \mathbf{1} \leq y+l$. Therefore, $y \geq f(y) \cdot \mathbf{1}-l$ and $f(y) \geq f(y)+f(-l)$. Then $f(-l) \leq 0(I)$. Since $f(x) \geq S_{l, d}(x), \forall x \in X$ so $f(-l+d \cdot \mathbf{1}) \geq S_{l, d}(-l+d \cdot \mathbf{1})$. Therefore, $f(-l)+d \geq \min \left\{\varphi_{l}(-l+d \cdot \mathbf{1}), d\right\}=d$. This implies that $f(-l) \geq 0(I I)$ by using $(I),(I I), f(-l)=0$ and $(l, d) \in D$.

Conversely, if $(l, d) \in D$, there exists $x \in X$ such that $S_{l, d}(x)>f(x)$ which implies that there exists $r>0$ such that $S_{l, d}(x)>f(x)+r$, and so $x>(f(x)+r) \cdot \mathbf{1}-l$. Since $f$ is topical and $f(-l)=0$. It shows that

$$
f(x)>f(x)+r+f(-l),
$$

which is a contradiction by choosing of $r$. Therefore, $S_{l, d}(x) \leq f(x), \forall x \in X$. Also $S_{l, d}(y) \leq f(y)$. Since $(l, d) \in D$, then $f(y) \leq S_{l, d}(y)$. It implies $f(y)=$ $S_{l, d}(y)$. Hence, $(l, d) \in \partial_{X_{s}} f(y)$. If $f(y) \cdot \mathbf{1}-y, d=|f(y)|$ or $d=f(y)$, then $(l, d) \in \partial_{X_{s}} f(y)$

$$
S_{l, d}(y)=\min \left\{\varphi_{l}(y), d\right\}=f(y) \text { and } f(y-f(y) \cdot \mathbf{1})=0 .
$$

Then $(l, d) \in \partial_{X_{s}} f(y)$.

It is worth noting that the function $S_{l, d}$ defined by remark (2) is sub-topical and by theorem (3.5) Lipschitz continuous. We now give characterizations of downward sets in terms of separation from outside points.

Theorem 3.8. Let $W \subseteq X$ and $S_{l, d}$ be a function defined by remark (2). Then the following assertions are equivalent:

i) $W$ is a downward set.

ii) For each $x \in X \backslash W$, there exists $(l, d) \in X \times \mathbb{R}_{+}$such that $S_{l, d}(w)<0 \leq$ $S_{l, d}(x)$. 


\section{Proof.}

$(i) \Longrightarrow(i i)$. Suppose that $(i)$ holds and $x \notin W$. Let $l=-x, d \in \mathbb{R}_{+}$, then by [6], $\varphi_{l}(w)<0 \leq \varphi_{l}(x) \forall w \in W$. From the definition of $S_{l, d}(w)=\min \left\{\varphi_{l}(w), d\right\}$,

$$
S_{l, d}(w)<0 \leq S_{l, d}(x)(\forall w \in W) .
$$

$($ ii $) \Longrightarrow(i)$. Suppose that $(i i)$ holds and $W$ is not a downward set. There is $w_{0} \in W$ and $x_{0} \in X \backslash W$ with $x_{0} \leq w_{0}$. By hypothesis, there exists $l \in X, d \in \mathbb{R}^{+}$ such that

$$
S_{l, d}(w)<0 \leq S_{l, d}\left(x_{0}\right)(\forall w \in W) .
$$

Since $S_{l, d}$ is increasing, then $S_{l, d}\left(x_{0}\right) \leq S_{l, d}\left(w_{0}\right)$.

Therefore,

$$
S_{l, d}\left(w_{0}\right)<0 \leq S_{l, d}\left(x_{0}\right) \leq S_{l, d}\left(w_{0}\right)
$$

This is a contradiction.

Theorem 3.9. Let $W \subseteq X$, and $S_{l, d}$ be the function defined by remark (2). Then the following assertions are equivalent:

i) $W$ is a closed downward subset of $X$.

ii) $W$ is downward, and for each $x \in X$ the set

$$
H=\{\lambda \in \mathbb{R}: x+\lambda \cdot 1 \in W\},
$$

is closed in $\mathbb{R}$.

iii) For each $x \in X \backslash W$. There is $(l, d) \in X \times \mathbb{R}_{++}$such that

$$
S_{l, d}(w)<0<S_{l, d}(x), \quad(w \in W) .(28)
$$

vi) For each $x \in X \backslash W$ there exists $(l, d) \in X \times \mathbb{R}_{++}$such that

$$
\sup _{w \in W} S_{l, d}(w)<S_{l, d}(x) \text {. }
$$

\section{Proof.}

$(i) \Longrightarrow(i i)$. The proof is the same as in [6]

(ii) $\Longrightarrow($ iii $)$. Suppose that $(i i)$ holds and $x \in X \backslash W$ is arbitrary. There is $l \in X$ such that

$$
\varphi(w, l)<0<\varphi(x, l)(\forall w \in W) .
$$

Let $d=\varphi(x, l) \in \mathbb{R}_{++}$, then

$$
S_{l, d}(w)=\min \{\varphi(w, l), d\}=\varphi(w, l)<0(\forall w \in W) .
$$

and

$$
S_{l, d}(x)=\min \{\varphi(x, l), d\}=\varphi(x, l)>0 .
$$

Therefore,

$$
S_{l, d}(w)<0<S_{l, d}(x)(\forall w \in W) .
$$

$($ iii $) \Longrightarrow(v i)$, is obvious.

$(v i) \Longrightarrow(i)$. Suppose that $(v i)$ holds and $W$ is not downward. There is $w_{0} \in W$ and $x_{0} \in X \backslash W$ with $x_{0} \leq w_{0}$. By hypothesis, there exists $l \in X, d \in \mathbb{R}_{++}$such that

$$
\sup _{w \in W} S_{l, d}(w)<S_{l, d}\left(x_{0}\right)
$$


Since $S_{l, d}(\cdot)$ is increasing, it follows that;

$$
S_{l, d}\left(x_{0}\right) \leq S_{l, d}\left(w_{0}\right) \leq \sup _{w \in W} S_{l, d}(w)<S_{l, d}\left(x_{0}\right) .
$$

This is a contraction. Hence, $W$ is a downward set. Finally, assume that $W$ is not closed. There is a sequence $\left\{w_{n}\right\}_{n \geq 1} \subseteq W$ and $x_{0} \in X \backslash W$ such that $\left\|w_{n}-x_{0}\right\| \longrightarrow 0$ as $n \longrightarrow \infty$. Since $x_{0} \in X \backslash W$, there exists $l \in X, d \in \mathbb{R}_{++}$ such that;

$$
\sup _{w \in W} S_{l, d}(w)<S_{l, d}\left(x_{0}\right)
$$

Thus,

$$
S_{l, d}\left(w_{n}\right) \leq \sup _{w \in W} S_{l, d}(w)(\forall n \geq 1) .
$$

From continuity of $S_{l, d}(\cdot), S_{l, d}\left(x_{0}\right) \leq \sup _{w \in W} S_{l, d}(w)$. This is a contradiction.

Lemma 3.10. Let $W$ be a closed downward subset of $X, w_{0} \in b d W$ and let $S_{l, d}$ be the function defined by remark (2). Then $S_{-w_{0}, d}(w) \leq 0(\forall w \in W$,$) and d \in \mathbb{R}_{+}$.

Proof. Suppose that this condition holds, by (see [6]), $\varphi\left(w,-w_{0}\right) \leq 0(\forall w \in W)$. Therefore, if $d \in \mathbb{R}_{+}$,

$$
S_{-w_{0}, d}(w)=\min \left\{\varphi\left(w,-w_{0}\right), d\right\}=\varphi\left(w,-w_{0}\right) \leq 0(\forall w \in W) .
$$

Lemma 3.11. Let $W$ be a closed downward subset of $X, w_{0} \in b d W, l=-w_{0}$ and $d \in \mathbb{R}_{+}$. Let $S_{l, d}$ be the function defined by remark (2). Then

$$
S_{l, d}(w) \leq 0=S_{l, d}\left(w_{0}\right),(\forall w \in W) .
$$

Proof. By hypothesis and [6]

$$
\varphi(w, l) \leq 0=\varphi\left(w_{0}, l\right)(\forall w \in W) .
$$

Let $d=0$

and

$$
S_{l, d}(w)=\min \{\varphi(w, l), d\}=\varphi(w, l) \leq 0,
$$

Therefore,

$$
S_{l, d}\left(w_{0}\right)=\min \left\{\varphi\left(w_{0}, l\right), d\right\}=0 .
$$

$$
S_{l, d}(w) \leq 0=S_{l, d}\left(w_{0}\right)(\forall w \in W)
$$

The following theorem gives a necessary and sufficient condition for the best approximation in terms of separation from outside points.

Theorem 3.12. Let $W$ be a closed downward subset of $X$ and $x_{0} \in X$. Let $y_{0} \in W$ and $r_{0}=\left\|x_{0}-y_{0}\right\|$. Assume that $S_{l, d}$ is the function defined by remark (2). Then the following assertions are equivalent:

i) $y_{0} \in P_{W}\left(x_{0}\right)$.

ii) There exists $l \in X$ and $d \in \mathbb{R}_{+}$such that;

$$
S_{l, d}(w) \leq 0 \leq S_{l, d}(y)\left(\forall w \in W, y \in B\left(x_{0}, r_{0}\right)\right)
$$

Moreover, if (30) holds with $l=-y_{0}$, then $y_{0}=\min P_{W}\left(x_{0}\right)$. 
Proof. $(i) \Longrightarrow(i i)$. Suppose that $y_{0} \in P_{W}\left(x_{0}\right)$, then $r_{0}=\left\|x_{0}-y_{0}\right\|=d\left(W, x_{0}\right)$. Since $W$ is closed downward subset of $X$, then by (see [6]), that the least element $w_{0}=x_{0}-r_{0} \cdot 1$ of the set $P_{W}\left(x_{0}\right)$ exists. Let $l=-w_{0} \in X$. Then,

$$
\varphi(w, l) \leq 0 \leq \varphi(y, l)\left(\forall w \in W, y \in B\left(x_{0}, r_{0}\right)\right) .(31)
$$

Let $\varphi_{l}\left(x_{0}+r_{0} \cdot \mathbf{1}\right)=d$, then $d \in \mathbb{R}_{+}$. Indeed $\forall y \in B\left(x_{0}, r_{0}\right)$. By $(4), 0 \leq y \leq$ $x_{0}+r_{0} \cdot \mathbf{1}$, then by $(31), 0 \leq \varphi(y, l) \leq \varphi\left(x_{0}+r_{0} \cdot \mathbf{1}, l\right)$. Therefore,

$$
S_{l, d}(w)=\min \{\varphi(w, l), d\}=\varphi(w, l) \leq 0,(\forall w \in W)
$$

and

$$
S_{l, d}(y)=\min \{\varphi(y, l), d\}=\varphi(y, l) \geq 0 .\left(\forall y \in B\left(x_{0}, r_{0}\right)\right)
$$

$($ ii $) \Longrightarrow(i)$. Assume that there exists $l \in X$ and $d \in \mathbb{R}_{+}$such that

$$
S_{l, d}(w) \leq 0 \leq S_{l, d}(y) .\left(\forall w \in W, y \in B\left(x_{0}, r_{0}\right)\right)
$$

From (4), $x_{0}-r_{0} \cdot \mathbf{1} \in B\left(x_{0}, r_{0}\right)$. From the hypothesis $S_{l, d}\left(x_{0}-r_{0} \cdot \mathbf{1}\right) \geq 0$. According to the definition of $S_{l, d}, \varphi\left(x_{0}-r_{0} \cdot \mathbf{1}, l\right) \geq 0$. Indeed $S_{l, d}\left(x_{0}-r_{0} \cdot \mathbf{1}\right)=$ $\min \left\{\varphi\left(x_{0}-r_{0} \cdot \mathbf{1}, l\right)\right\} \geq 0$. Since $\varphi(., l)$ is topical, $\varphi\left(x_{0}, l\right) \geq r_{0}$. Due to $(7)$,

$$
r_{0} \cdot \mathbf{1} \leq \varphi\left(x_{0}, l\right) \cdot \mathbf{1} \leq x_{0}+l .
$$

Let $w \in W$ be an arbitrary and $p_{w}=\varphi\left(w,-x_{0}\right) \cdot \mathbf{1}+x_{0} \in X$. Then $\varphi\left(w,-x_{0}\right) \cdot \mathbf{1} \leq$ $w-x_{0}$ and $p_{w} \leq w$. Since $W$ is downward set and $w \in W$, it follows that $p_{w} \in W$. By hypothesis $S_{l, d}\left(p_{w}\right) \leq 0$ and since $d \in \mathbb{R}_{+}, \varphi\left(p_{w}, l\right) \leq 0$. Since $\varphi\left(p_{w},.\right)$ is topical and (32) holds,

$$
S_{-x_{0}, d}\left(p_{w}\right) \leq \varphi\left(p_{w},-x_{0}\right) \leq \varphi\left(p_{w}, l\right)-r_{0} \leq-r_{0} .
$$

Since $\varphi\left(.,-x_{0}\right)$ is topical

$$
-r_{0} \geq \varphi\left(p_{w},-x_{0}\right)=\varphi\left(\varphi\left(w,-x_{0}\right) \cdot \mathbf{1}+x_{0},-x_{0}\right)=\varphi\left(w,-x_{0}\right) .
$$

From Lipschitz continuity of $\varphi_{-x_{0}}$,

$$
r_{0} \leq\left|\varphi\left(w,-x_{0}\right)\right|=\left|\varphi\left(x_{0},-x_{0}\right)-\varphi\left(w,-x_{0}\right)\right| \leq\left\|x_{0}-w\right\| .
$$

Thus $r_{0} \leq\left\|x_{0}-w\right\|$ for all $(w \in W)$ and $\left\|x_{0}-y_{0}\right\|=d\left(x_{0}, W\right)$. Consequently, $y_{0} \in P_{W}\left(x_{0}\right)$. Finally, suppose that (30) holds with $l=-y_{0}$. From implication (ii) $\Longrightarrow(i), y_{0} \in P_{W}\left(x_{0}\right)$. Let $w \in P_{W}\left(x_{0}\right)$ be an arbitrary. Thus, $\left\|x_{0}-w\right\|=$ $d\left(x_{0}, W\right)=\left\|x_{0}-y_{0}\right\|=r_{0}$, that is $w \in B\left(x_{0}, r_{0}\right)$. It follows from the hypothesis $S_{-y_{0}, d}(w) \geq 0$ and so $0 \leq S_{-y_{0}, d}(w) \cdot \mathbf{1} \leq \varphi\left(w,-y_{0}\right) \cdot \mathbf{1} \leq w-y_{0}$. This implies that $y_{0} \leq w$ for all $w \in P_{W}\left(x_{0}\right)$. Hence, $y_{0}=\min P_{W}\left(x_{0}\right)$, which it completes the proof. 


\section{REFERENCES}

1. M. Alimohammady, A. Shahmari, Superlinear separation on downward sets and characterization of topical functions over ordered Banach spaces., Optimization, to appear.

2. J. Gunawardena, An introduction to idempotency. Idempotency (Bristol,1994), 1-49, publ. Newton Inst.,11, Cambridge Univ. Press, Cambridge, 1998.

3. J. Gunawardena, From max-plus algebra to nonexpansive mappings: a nonlinear theory for discrete event systems, Theoretical Computer Science, to appear (revised version of technical Report HPL-BRIMS-99-07, Hewlett-Packard Labs, 1999).

4. J.-E. Martinez-Legaz, A. M. Rubinov, and I. Singer, Downward sets and thir separeation and approximation properties, J. Global Optimization 23 (2002), 111-137.

5. H. Mohebi, Topical functions and their properties in a class of ordered Banach spaces, J. Math. Anal. Appl. in press. 1.1

6. H. Mohebi, ,Rubinov,A. M. Best approximation by downward sets with applications, J. Math. Anal. Appl. in press. 1.1, 2.11

7. R.T. Rockafellar, Convex Analysis, Princeton Mathematical series, No. 28, Princeton University press, Princeton, N.J., 1970.

8. A. M. Rubinov, Abstract convexity and global optimization, Kluwer Academic Publishers, Boston, Dordrech, London, 2000.

9. A. M. Rubinov, and I. Singer, Topical and sub-topical functions, downward sets and abstract convexity, Optimization , 50 (2001), 307-351.2.1

1,2 Department of Mathematics, University of Mazandaran, Babolsar 47416 1468, IRAN.

E-mail address: amohsen@umz.ac.ir

${ }^{2}$ Islamic Azad University, Ayatollah Amoli Branch, Amol, Iran

E-mail address: ashahmari@yahoo.com 